\title{
The impacts of new energy vehicles on fleet average oil consumption of passenger vehicles in China
}

\author{
Jiacai $\mathrm{Li}^{*}$ \\ Law School, Shantou University, 515063 Shantou Guangdong, China
}

\begin{abstract}
Using authoritative data and calculation formulas, this paper reveals exactly the role of new energy vehicles (NEV) in the decrease of fleet average oil consumption (FAOC) of passenger vehicles in China. NEV is the reason for the gap among the accounted FAOC, the real FAOC of passenger vehicles and the FAOC of conventional energy vehicles (CEV). Specifically, the NEV multipliers result in the difference between the accounted FAOC and the real FAOC of passenger vehicles, while the low oil consumption and output of NEV result in the difference between the real FAOC of passenger vehicles and the FAOC of CEV. NEV is accelerating the reduction of the FAOC of passenger vehicles in China.

Keywords: Passenger vehicle; Fleet Average Oil Consumption (FAOC); New Energy Vehicle (NEV).
\end{abstract}

\section{Introduction}

According to China's corporate average fuel consumption (CAFC ) standard for passenger vehicles, since 2016, NEV will be included in the calculation of actual CAFC; Furthermore, NEV output can be counted by a multiplier greater than 1 and its non-oil fuel consumption is excluded (Table 1) [1]. NEV includes battery electric vehicles (BEV), plugin hybrid electric vehicles (PHEV) and fuel cell vehicles (FCV). Therefore, the so-called "actual FAOC of passenger vehicles" is actually the accounted FAOC of passenger vehicles, rather than the real FAOC of passenger vehicles, let alone the real fleet average fuel consumption (including non-oil fuel consumption) of passenger vehicles.

Table 1. NEV's preferences in actual CAFC calculation.

\begin{tabular}{l|c|c|c|c|c}
\hline Year & 2016 & 2017 & 2018 & 2019 & 2020 \\
\hline NEV Multiplier & 5 & 5 & 3 & 3 & 2 \\
\hline $\begin{array}{l}\text { Favor for NEV's fuel } \\
\text { consumption counting }\end{array}$ & $\begin{array}{l}\text { Only is PHEV's oil consumption counted (excluding electricity } \\
\text { consumption of BEV and PHEV, and hydrogen consumption of FCV) }\end{array}$ \\
\hline
\end{tabular}

\footnotetext{
* Corresponding author: jcli@stu.edu.cn
} 
Using official data and simple algorithms, this paper calculates the real FAOC of China's passenger vehicles, the FAOC of NEV as passenger vehicles and the FAOC of CEV as passenger vehicles. This paper reveals accurately the role of NEV in the decrease of FAOC for passenger vehicles in China.

\section{The FAOC of passenger vehicles (2016-2019)}

With FTOC, FTOC $C_{1}$ and $F T O C_{2}$ denoting FAOC of passenger vehicles, the overall oil consumption of CEV and the overall oil consumption of PHEV (the overall oil consumption of NEV); with $F A O C_{1}$ and FAOC 2 denoting FAOC of CEV and FAOC of PHEV (FAOC of $\mathrm{NEV}$ ); with $Y, Y_{l}$ and $Y_{2}$ denoting output of passenger vehicles, output of CEV and output of $\mathrm{NEV}$, then FTOC equals the product of FAOC and $Y, F T O C_{1}$ equals the product of $F A O C_{1}$ and $Y_{1}, F T O C_{2}$ is equal to $F A O C_{2}$ times $Y 2$, and FTOC is equal to $F T O C_{1}$ plus $F T O C_{2}$. Then $m$ represents the NEV multiplier, so the preferences of NEV in the calculation of actual CAFC means that Formula 1 can be used to calculate the accounted FAOC of passenger vehicles $\left(F A O C^{A}\right)$.

$$
F A O C^{A}=\frac{F T O C_{1}+F T O C_{2}}{Y_{1}+m Y_{2}}=\frac{F T O C}{Y_{1}+m Y_{2}}
$$

All data in Table 2 are from the official website of the Ministry of Industry and Information Technology (MIIT), which shows the rapid downward trend of $F A O C^{A}$ from 2016 to $2019^{[2]}$.

Table 2. Output (million) and accounted $F A O C(\mathrm{~L} / 100 \mathrm{~km})$ of passenger vehicles.

\begin{tabular}{c|c|c|c|c}
\hline Year & $Y$ & $Y_{1}$ & $Y_{2}$ & FAOC $^{A}$ \\
\hline 2016 & 24.4947 & 24.1673 & 0.3274 & 6.43 \\
\hline 2017 & 24.6929 & 24.1121 & 0.5808 & 6.05 \\
\hline 2018 & 23.1391 & 22.1039 & 1.0352 & 5.80 \\
\hline 2019 & 20.93 & 19.8536 & 1.0764 & 5.56 \\
\hline
\end{tabular}

\section{The real FAOC of passenger vehicles}

The real FAOC of passenger vehicles $\left(F A O C^{R}\right)$ is equal to the total oil consumption of passenger vehicles divided by the total production of the passenger vehicles (Formula 2).

$$
F A O C^{R}=\frac{F T O C_{1}+F T O C_{2}}{Y_{1}+Y_{2}}=\frac{F T O C}{Y_{1}+Y_{2}}
$$

According to Formula 1 and 2, Formula 3 and 4 can be deduced.

$$
\begin{gathered}
F T O C=F_{A O C}^{A}\left(Y_{1}+m Y_{2}\right)=F_{A O C}^{R}\left(Y_{1}+Y_{2}\right) \\
F A O C^{R}=\frac{Y_{1}+m Y_{2}}{Y_{1}+Y_{2}} F A O C^{A}
\end{gathered}
$$

According to Table 1, 2 and Formula 4, the real FAOC of passenger vehicles from 2016 to 2019 can be calculated (Table 3).

Table 3. The real FAOC of passenger vehicles (L/100 km).

\begin{tabular}{c|c|c|c|c}
\hline Year & 2016 & 2017 & 2018 & 2019 \\
\hline$F^{\prime} A O C^{R}$ & 6.77 & 6.62 & 6.32 & 6.13 \\
\hline
\end{tabular}




\section{The FAOC of NEV (2016-2019)}

The FTOC of NEV is equal to the FTOC of PHEV. When the total PHEV production and PHEV's FAOC are known, the FAOC of NEV can be calculated (Formula 5).

$$
F A O C_{2}=\frac{F T O C_{2}}{Y_{2}}
$$

The "PHEV Sales" in Table 4 is used as a substitute for "PHEV Production" (the latter data are not available and the gap between them is very small) ${ }^{[3]}$. The FAOC of PHEV in Table 4 is estimated based on the FAOC of BYD Motor Company's PHEV (BYD's PHEV sales account for approximately $25 \%$ of total PHEV sales).

The FAOC of NEV $\left(\mathrm{FAOC}_{2}\right)$ is shown in Table 4.

Table 4. FTOC and FAOC of NEV.

\begin{tabular}{c|c|c|c|c}
\hline Year & $\begin{array}{c}\text { PHEV Sales } \\
\text { (thousand) }\end{array}$ & $\begin{array}{c}\text { FAOC of PHEV } \\
(\mathrm{L} / 100 \mathrm{~km})\end{array}$ & $\begin{array}{c}\text { FTOC } 2 \\
(1000 \mathrm{~L} / 100 \mathrm{~km})\end{array}$ & $\begin{array}{c}\text { FAOC }_{2} \\
(\mathrm{~L} / 100 \mathrm{~km})\end{array}$ \\
\hline 2016 & 80.562 & 1.6 & 128.899 & 0.39 \\
\hline 2017 & 106.850 & 1.6 & 170.960 & 0.29 \\
\hline 2018 & 241.351 & 1.5 & 362.027 & 0.35 \\
\hline 2019 & 202.180 & 1.5 & 303.270 & 0.28 \\
\hline
\end{tabular}

\section{The FAOC of CEV}

According to Formula 4 and Table 1, 2, 3 and 4, the FAOC of CEV can be calculated (Formula 6 and Table 5).

$$
F A O C_{1}=\frac{F T O C-F T O C_{2}}{Y_{1}}
$$

Table 5. FAOC of CEV (L/100 km).

\begin{tabular}{c|c|c|c|c}
\hline Year & 2016 & 2017 & 2018 & 2019 \\
\hline$F A O C_{1}$ & 6.86 & 6.77 & 6.6 & 6.45 \\
\hline
\end{tabular}

Figure 1 shows four types of FAOC of passenger vehicles in 2016-2019.

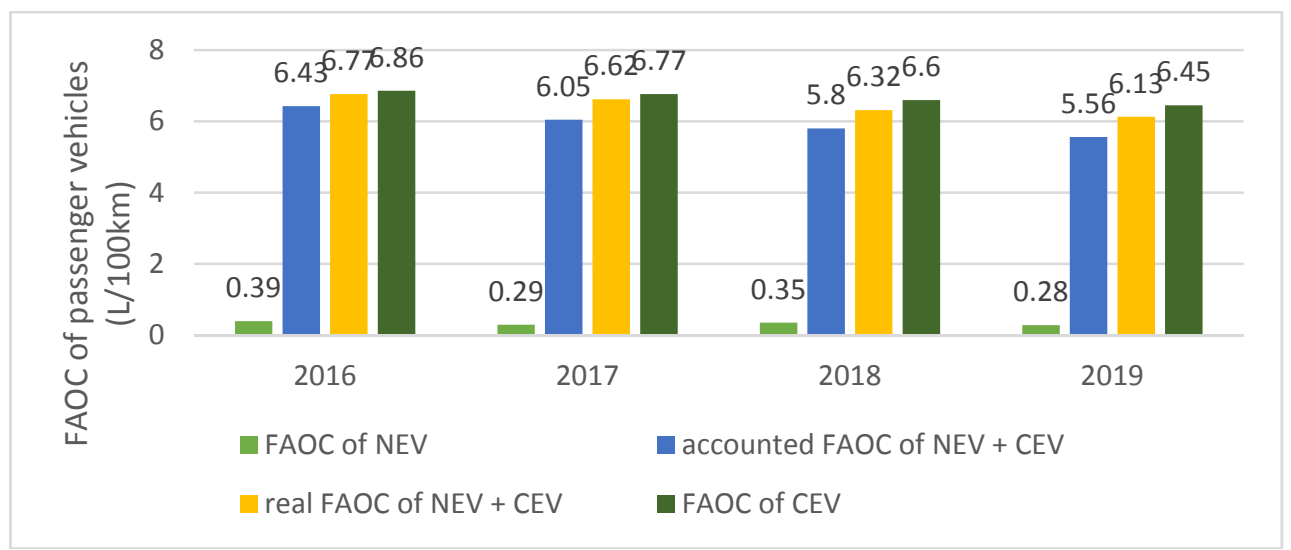

Fig. 1. Four types of FAOC for passenger vehicles. 


\section{The impacts of NEV multipliers on FAOC of passenger vehicles}

By comparing Formula 1 and Formula 2, it can be found that the difference between the accounted and the real $F A O C$ of passenger vehicles comes from the impact of NEV multiplier. Thus, FAOC ${ }^{A}-F_{A O C^{R}}$ can represents the decrease in $F A O C$ of passenger vehicles due to NEV multiplier (Table 6).

Table 6. FAOC reductions of passenger vehicles caused by NEV multipliers (L/100 km).

\begin{tabular}{c|c|c|c|c}
\hline Year & 2016 & 2017 & 2018 & 2019 \\
\hline$F^{A}-F A O C^{R}$ & -0.34 & -0.57 & -0.52 & -0.57 \\
\hline
\end{tabular}

\section{The impacts of low fuel consumption and output of NEV on FAOC}

By comparing Formula 2 and Formula 6, it can be found that the difference between the real $F A O C$ of passenger vehicles and the $F A O C$ of $\mathrm{CEV}$ comes from low fuel consumption and output of NEV. Thus, $F A O C^{R}-F A O C_{1}$ can represents the decrease in $F A O C$ of passenger vehicles due to low fuel consumption and output of NEV (Table 7).

Table 7. FAOC reductions caused by low fuel consumption and output of NEV (L/100 km).

\begin{tabular}{c|c|c|c|c}
\hline Year & 2016 & 2017 & 2018 & 2019 \\
\hline$F^{R}-F^{R} C_{1}$ & -0.09 & -0.15 & -0.28 & -0.32 \\
\hline
\end{tabular}

\section{The total impacts of NEV on FAOC}

The total effect of NEV on $F A O C$ of passenger vehicles is equal to $F A O C^{A}-F A O C_{1}$, or to the sum of the two effects mentioned above (Table 8).

Table 8. Total decreases in FAOC of passenger vehicles caused by NEV (L/100 km).

\begin{tabular}{c|c|c|c|c}
\hline Year & 2016 & 2017 & 2018 & 2019 \\
\hline FAOC $^{A}-F_{A O C_{1}}$ & -0.43 & -0.72 & -0.8 & -0.89 \\
\hline
\end{tabular}

Figure 2 shows the decreases of $F A O C$ of passenger vehicles caused by NEV.

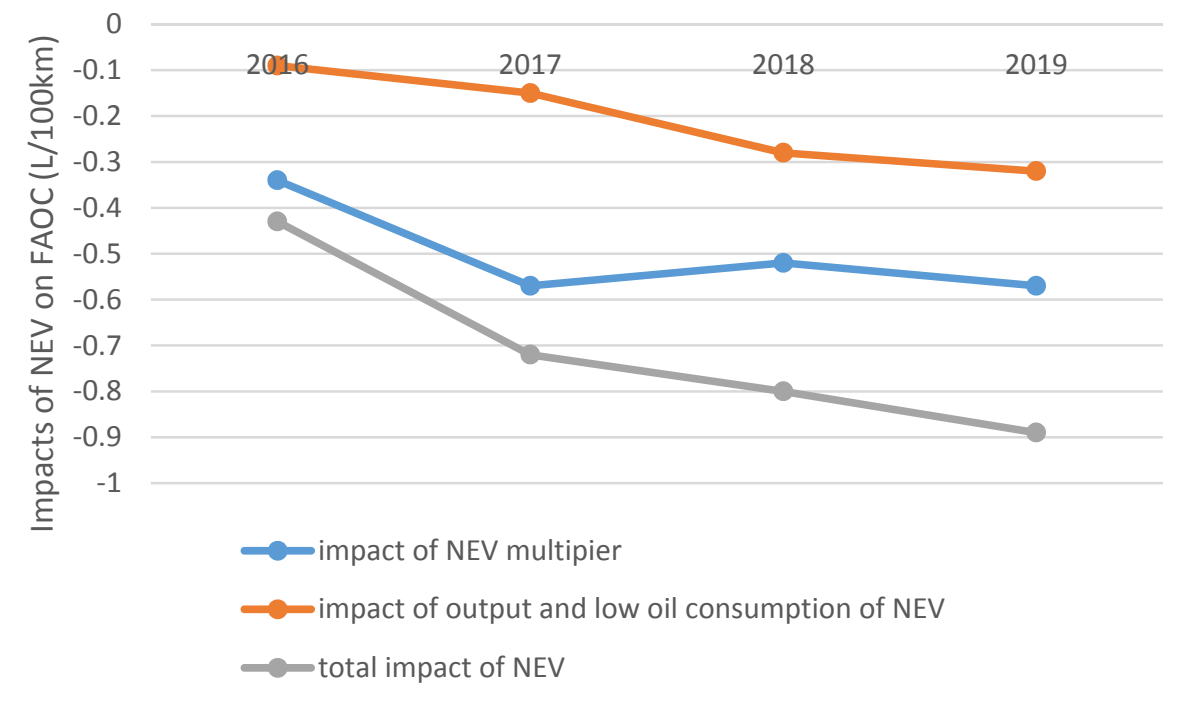

Fig. 2. FAOC reductions of passenger vehicles caused by NEV. 


\section{Conclusions}

In each year from 2016 to 2019, the FAOC of NEV, the accounted FAOC of passenger vehicles, the real FAOC of passenger vehicles and the FAOC of CEV increase in turn. The differences between the accounted and the real FAOC come from the impacts of NEV multipliers, while the differences between the real FAOC of passenger vehicles and the FAOC of CEV come from low oil consumption and output of NEV. The cumulative effect of NEV on the $F A O C$ of passenger vehicles is equal to the sum of the above two effects, and this total effect shows a trend of gradual increase.

\section{Acknowledgement}

The author thanks the university-strengthening project by innovation of Shantou University for providing funding for this study.

\section{References}

1. General administration of quality supervision, inspection, and quarantine of China (GAQSIQ), Standardization administration of China (SAC). Fuel consumption evaluation methods and targets for passenger vehicles: GB 2799-2014[S] (in Chinese). Beijing, China Standard Publishing House, 2015.

2. Ministry of industry and information technology (MIIT) of China, et al. Bulletin on China's CAFC credits and NEV credits of passenger vehicles in 2016, 2017, 2018, 2019 (in Chinese). [EB/OL]. http://www.miit.gov.cn/, 2017, 2018, 2019, 2020.

3. China's Passenger Car Market Information Conference. PHEV sales in 2016-2019 (in Chinese) [DB/OL]. http://biz.touchev.com/industry_data, 2020. 